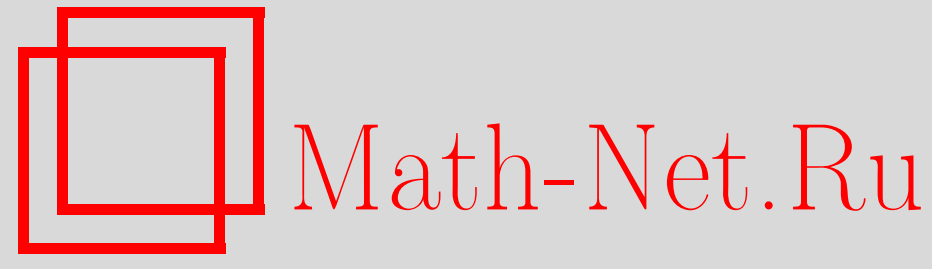

Общероссийский математический портал

К. М. Эминян, Об одной бинарной задаче, Матем. заметки, 1996, том 60, выпуск 4, 634-637

DOI: https://doi.org/10.4213/mzm1877 
Использование Общероссийского математического портала MathNet.Ru подразумевает, что вы прочитали и согласны с пользовательским соглашением

http://www . mathnet.ru/rus/agreement

Параметры загрузки:

IP : 3.85 .5 .30

26 апреля 2023 г., 09:57:33

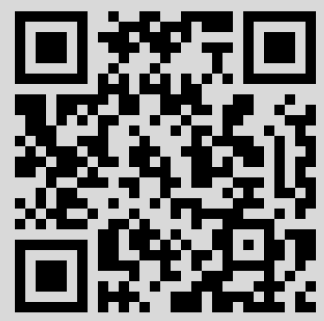




\section{ОБ ОДНОЙ БИНАРНОЙ ЗАДАЧЕ}

\section{К. М. Эминян}

Рассмотрим двоичное разложение натураљного числа $n$ :

$$
n=\sum_{i=0}^{\infty} \varepsilon_{i} 2^{i}
$$

где $\varepsilon_{k}=0,1, k=0,1,2, \ldots$ Пусть

$$
\mathbb{N}_{0}=\left\{n: n \in \mathbb{N}, \sum_{i=0}^{\infty} \varepsilon_{i} \equiv 0(\bmod 2)\right\}, \quad \mathbb{N}_{1}=\mathbb{N} \backslash \mathbb{N}_{0} .
$$

Обозначим

$$
\chi(n, m ; a)=\left\{\begin{array}{l}
1, \text { если } n \equiv a(\bmod a), \\
0, \text { если } n \neq \equiv(\bmod a) .
\end{array}\right.
$$

А. О. Гельфонд доказал (см. [1]) теорему о том, что

$$
\sum_{n \leqslant X, n \in \mathbb{N}} \chi(n, m ; a)=\frac{X}{2 m}+O\left(X^{\lambda}\right),
$$

где $\lambda=\log 3 / 2 \log 2$.

Работавыполнена при финансовой поддержке Международного научного фонда, грант NFV000. 
В статье [2] получена асимптотическая формула со степенным понижением в проблеме делителей для случая, когда суммирование распространяется на так назьваемые "позиционные последовательности $\left(\mathbb{N}_{0}, \mathbb{N}_{1}\right)$ натуральных чисел.

В этой статье рассматривается другой тип задачи, связанный с "позиционными последовательностями".

Обозначим через $F_{i, j}(X)$ число решений уравнения $n-m=1, n, m \leqslant X$, $n \in \mathbb{N}_{i}, m \in \mathbb{N}_{j}, i, j=0,1$.

ТеоремА. Справедливы следующие асимптотические формуль:

$$
\begin{array}{ll}
F_{0,0}(X)=\frac{X}{6}+O(\log X), & F_{1,1}(X)=\frac{X}{6}+O(\log X), \\
F_{1,0}(X)=\frac{X}{3}+O(\log X), & F_{0,1}(X)=\frac{X}{3}+O(\log X) .
\end{array}
$$

Сначала докажем следующую лемму.

Лемма. Справедлива формула

$$
S_{0}(X)=\sum_{n \leqslant X} \varepsilon(n) \varepsilon(n+1)=-\frac{X}{3}+O(\log X),
$$

əде

$$
\varepsilon(n)=\left\{\begin{aligned}
1, & \text { если } n \in \mathbb{N}_{0}, \\
-1, & \text { если } n \in \mathbb{N}_{1} .
\end{aligned}\right.
$$

ДокАЗАТЕЛЬСтво. Рассмотрим сумму

$$
S_{r}(X)=\sum_{k \leqslant\left(X-2^{r}+1\right) / 2^{r}} \varepsilon(k) \varepsilon(k+1) .
$$

Перепишем $S_{r}(X)$ таким образом:

$$
\begin{aligned}
& S_{r}(X)=\sum_{k=2 n \leqslant\left(X-2^{r}+1\right) / 2^{r}} \varepsilon(k) \varepsilon(k+1)+\sum_{k=2 n+1 \leqslant\left(X-2^{r}+1\right) / 2^{r}} \varepsilon(k) \varepsilon(k+1) \\
& =\sum_{n \leqslant\left(X-2^{r}+1\right) / 2^{r+1}} \varepsilon(2 n) \varepsilon(2 n+1)+\sum_{n \leqslant\left(X-2^{r+1}+1\right) / 2^{r+1}} \varepsilon(2 n+1) \varepsilon(2(n+1)) \\
& =A+B .
\end{aligned}
$$

Заметим, что $\varepsilon(n)=\varepsilon(2 n)$ и $\varepsilon(2 n+1)=-\varepsilon(n)$. Действительно, пусть

$$
n=\sum_{i=0}^{N(n)} \varepsilon_{i} 2^{i}
$$

тогда

$$
2 n=\sum_{i=0}^{N(n)} \varepsilon_{i} 2^{i+1}
$$


и, следовательно, количество единиц в двоичном разложении чисел $n$ и $2 n$ одинаковы. В силу определения функции $\varepsilon(n)$ это означает, что $\varepsilon(n)=\varepsilon(2 n)$. А для числа $2 n+1$ имеем представление

$$
1+\sum_{i=0}^{N(n)} \varepsilon_{i} 2^{i+1}
$$

т.е. количество разрядов в двоичном разложении числа $2 n+1$ на единицу болшше, чем у числа $n$, поэтому $\varepsilon(2 n+1)=-\varepsilon(n)$. Следовательно,

$$
\begin{aligned}
A & =\sum_{n \leqslant\left(X-2^{r}+1\right) / 2^{r+1}} \varepsilon(n) \varepsilon(2 n+1)=-\left[\frac{X-2^{r}+1}{2^{r+1}}\right], \\
B & =\sum_{n \leqslant\left(X-2^{r+1}+1\right) / 2^{r+1}} \varepsilon(2 n+1) \varepsilon(2(n+1)) \\
& =-\sum_{n \leqslant\left(X-2^{r+1}+1\right) / 2^{r+1}} \varepsilon(n) \varepsilon(n+1)=-S_{r+1}(X) .
\end{aligned}
$$

Получаем следующую рекуррентную формулу:

$$
S_{r}(X)=-\left[\frac{X-\left(2^{r}-1\right)}{2^{r+1}}\right]-S_{r+1}(X) .
$$

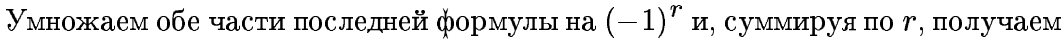

$$
\sum_{r=0}^{N}(-1)^{r} S_{r}(X)=\sum_{r=0}^{N}(-1)^{r+1}\left[\frac{X-\left(2^{r}-1\right)}{2^{r+1}}\right]+\sum_{r=0}^{N}(-1)^{r+1} S_{r+1}(X),
$$

где $N=\left[\log _{2}((X+1) / 2)\right]$. Следовательно,

$$
S_{0}(X)=\sum_{r=0}^{N}(-1)^{r+1}\left[\frac{X-\left(2^{r}-1\right)}{2^{r+1}}\right]+O(1) .
$$

Поэтому

$$
\sum_{n \leqslant X} \varepsilon(n) \varepsilon(n+1)=S_{0}(X)=-\frac{X}{3}+O(\log X),
$$

Лемма доказана.

ДОКАЗАТЕЛЬСТВО ТЕОРЕМЫ. Ввиду определения $\varepsilon(n)$ имеем

$$
F_{0,0}(X)=\sum_{\substack{n, m \leqslant X \\ n-m=1}} \frac{1+\varepsilon(n)}{2} \frac{1+\varepsilon(m)}{2}
$$

или

$$
F_{0,0}(X)=\frac{1}{4} F(X)+\frac{1}{4} G_{1}(X)+\frac{1}{4} G_{2}(X)+\frac{1}{4} G_{3}(X),
$$


где $F(X)$ - число решений уравнения $n-m=1, m, n \leqslant X$ (ясно, что $F(X)=$ $X+O(1))$, и

$$
\begin{aligned}
G_{1}(X) & =\sum_{\substack{n, m \leqslant X \\
n-m=1}} \varepsilon(n)=\sum_{n \leqslant X} \varepsilon(n) \sum_{\substack{m \leqslant X \\
n-m=1}} 1, \\
G_{2}(X) & =\sum_{\substack{n, m \leqslant X \\
n-m=1}} \varepsilon(m)=\sum_{m \leqslant X} \varepsilon(m) \sum_{\substack{n \leqslant X \\
n-m=1}} 1, \\
G_{3}(X) & =\sum_{\substack{n, m \leqslant X \\
n-m=1}} \varepsilon(n) \varepsilon(m)=\sum_{n \leqslant X} \varepsilon(n) \varepsilon(n+1) .
\end{aligned}
$$

По лемме $S_{0}(X)=G_{3}(X)$.

Заметим, что при фиксированном $n$ или $m$ уравнение $n-m=1, n, m \leqslant X$, может иметь одно решение, поэтому

$$
G_{1}(X)=\sum_{n<X} \varepsilon(n)+O(1), \quad G_{2}(X)=\sum_{m<X} \varepsilon(m)+O(1),
$$

HO

$$
\begin{aligned}
\sum_{n<X} \varepsilon(n)= & \sum_{n=2 t<X} \varepsilon(n)+\sum_{n=2 t+1<X} \varepsilon(n) \\
= & \sum_{t<X / 2} \varepsilon(2 t)+\sum_{t<(X-1) / 2} \varepsilon(2 t+1)=\sum_{t<X / 2} \varepsilon(t)-\sum_{t<(X-1) / 2} \varepsilon(t)=O(1) .
\end{aligned}
$$

Аналогичньгм образом $G_{2}(X)=O(1)$. Поэтому

$$
F_{0,0}(X)=\frac{1}{4} X+\frac{1}{4} S_{0}(X)+O(1)=\frac{1}{4} X-\frac{X}{3} \cdot \frac{1}{4}+O(\log X)=\frac{X}{6}+O(\log X) .
$$

Соответственно

$$
\begin{gathered}
F_{1,1}(X)=\frac{1}{4} X+\frac{1}{4} S_{0}(X)+O(1) \\
F_{1,0}(X)=\frac{1}{4} X-\frac{1}{4} S_{0}(X)+O(1), \quad F_{0,1}(X)=\frac{1}{4} X-\frac{1}{4} S_{0}(X)+O(1) .
\end{gathered}
$$

Следовательно, в силу леммы теорема полностью доказана.

Поступило

21.07 .95

\section{СПИСОК ЦИТИРОВАННОЙ ЛИТЕРАТУРЫ}

1. Gelfond A. O. // Acta Arith. 1968. V. 13. P. 259-265. 2. Эминян К. М.// Изв. АН СССР. Сер. матем. 1991. Т. 55. № 3. С. 680-686. 